\title{
Evaluación de operaciones lógico-matemáticas mediante dos métodos distintos en niños del pueblo indígena Shipibo-Konibo*
}

\section{Assessment of mathematical operations by two different methods in Shi- pibo-Konibo indigenous children}

\author{
Jorge Villalba Garcés** y Susana Frisancho Hidalgo***
}

\begin{abstract}
*Trabajo realizado en el marco del proyecto Evaluación de competencias cognitivas y morales en niños y adolescentes de comunidades Shipibo (Ucayali) y percepción sobre la educación en sus comunidades (Proyecto de Investigación 2014-0008) y financiado por la Pontificia Universidad Católica del Perú. **Licenciado en Psicología Educacional. Miembro del Grupo de Investigación en Cognición,

Aprendizaje y Desarrollo del Departamento de Psicología y Pre-docente del curso Ética y

Responsabilidad Profesional de la especialidad de Psicología de la Pontificia Universidad Católica del Perú. E-Mail: jvillalba@pucp.pe

***Doctora en Psicología del Desarrollo, Magister en Psicología y Licenciada en Psicología

Educacional. Profesora Principal del Departamento de Psicología y Coordinadora del Grupo de Investigación en Cognición, Aprendizaje y Desarrollo del Departamento de Psicología de la Pontificia Universidad Católica del Perú. E-Mail: sfrisan@pucp.edu.pe http://www.pucp.edu.pe/susana-frisancho-hidalgo/
\end{abstract}

Los autores agradecen la colaboración de la comunidad Shipibo-Konibo de Bethel, y especialmente al Prof. Hilario Díaz Peña Docente de $5^{\circ}$ y $6^{\circ}$ grados de primaria, quien brindó todas las facilidades para realizar este estudio.

Pontificia Universidad Católica del Perú. Lima, Perú.

\section{Resumen}

Se presentan los resultados de una evaluación de las operaciones matemáticas de adición y sustracción, en un grupo de niños de enseñanza primaria (5 niñas y 2 niños) de 7, 9 y 11 años, de una comunidad nativa Shipibo-Konibo de la región Ucayali, en la Amazonía del Perú. Se realizó con dos métodos distintos, la Entrevista Clínico-crítica de Piaget, que incluyó problemas culturalmente contextualizados y material representativo y manipulable como figuras de animales y bolitas para armar collares, y una prueba tradicional de lápiz y papel. Ambas pruebas evaluaron el mismo tipo de operaciones, extraídas de lo que propone el diseño curricular nacional para esos grados. Los resultados muestran que los niños tienen dificultades tanto en la adición como en la sustracción y no logran resolver con éxito todas las tareas. Sin embargo, se pueden observar mejores resultados con la evaluación cualitativa por medio de la entrevista clínico-crítica, en contraste con la evaluación tradicional de lápiz y papel que evidencia resultados mucho más pobres. La primera otorga además mayor información sobre el proceso operativo de los niños y muestra que los niveles de desarrollo de sus competencias matemáticas van de la mano con lo que plantea la teoría piagetiana. Los resultados se discuten señalando la universalidad del conocimiento lógico-matemático y su pertinencia para comprender los procesos de aprendizaje en contextos de diversidad cultural y analizando críticamente el modo en que la evaluación constructivista ofrece mayor 
información y recursos para los docentes de educación intercultural bilingüe de comunidades amazónicas del Perú.

Palabras clave: Conocimiento lógico-matemático; Jean Piaget; Método clínico-crítico; Comunidades indígenas amazónicas; Educación intercultural.

\section{Abstract}

Elementary-school children from a ShipiboKonibo indigenous community in the Ucayali region, in the Amazonian rainforest of Perú, were evaluated regarding their abilities to solve addition and subtraction problems. These operations were assessed by two means: through Jean Piaget's clinical-critical method (using culturally contextualized problems and concrete materials such as pictures of Amazonian fish, pictures of arrows, or beads and thread to make necklaces), and by a traditional pencil-and-paper test. Both the clinical interview and the penciland-paper test evaluated the same type of operations, which were taken from the national curricular program for these school grades. The Shipibo-Konibo people are an Amazonian indigenous group that speaks a native language in the Panoan family; since most members of this group are fluent in Spanish, however, no translator was needed and the assessments were conducted in Spanish. The Shipibo-Konibo people are principally settled along the Ucayali River in the Amazon rainforest in Perú, although currently many of them have relocated to other areas of the country, including Lima the capital city, in search of better work or education opportunities. After the Asháninka and the Awajún, the Shipibo-Konibo is the third largest Amazonian indigenous group in Perú.

Informed consent was obtained following the guidelines of Frisancho, Delgado, and Lam (2015), which are based on previous experience working with Amazonian indigenous communities in the Ucayali region of Peru. As research has shown that individuals from cultural diverse backgrounds may have different expectations for the research process, and may perceive it in a different way than people from industrialized nations (Lakes, Vaughan, Jones, Burke, Baker, \& Swanson 2012), informed consent included both individual consent and a communitarian meeting. It also included the donation of gifts (tools, groceries, and other useful items) for the community, and a debriefing meeting with the community's school teachers.

The assessment was conducted by two researchers in a school classroom. It took around 30 minutes with the older children and 45 with the younger ones. In all cases the clinical-critical interview was applied first (addition and then subtraction), and finally the pencil-and-paper test. Although a native speaker of shipibo was present during the evaluation, his services were not needed as children were fluent in Spanish. Results show that children have difficulties in the development of both addition and subtraction. They make counting mistakes and have trouble understanding the logic of subtraction (taking a number from another, larger one). Some do not conceptualize subtraction as the opposite of addition and, in consequence, cannot foresee that joining two numbers that were previously separated will result in the same original quantity. Children aged seven were unable to solve any of the operations in the pencil-and-paper test. However, better results were obtained through qualitative, Piagetian assessments, in contrast to the quantitative, pencil-and-paper assessments. During the interviews, children were able to show their cognitive processes and ways of thinking while solving the problems, and with scaffolding, the use of concrete materials such as pictures or beads, and strategic help from the evaluators (Parrat, 2016a, 2016b), many of them were also able to develop a better comprehension of the problem and self-correct their initial answers. Developmental levels for addition and subtraction consistent with Piagetian theory were identified. These levels show a progression from the impossibility of grasping logical addition or subtraction, to the capabilities of psychological reversibility and logical composition of inverse and direct operations. The results are discussed using Piaget's theory and the problem of particulars and universals in cognitive development, and analyzing critically how this kind of assessment can help elementary school teachers respond to the needs of intercultural bi- 
lingual education in Peru's Amazonian indigenous communities.

Key words: Logical-mathematics knowledge; Jean Piaget; Clinical-critic method; Amazonian indigenous communities; Intercultural education.

\section{Introducción}

Aunque en América Latina existen diferencias entre países con respecto al aprendizaje de la Matemática (Cáceres, De la Peña, Pineda, Di Prisco \& Solotar, 2014; Murillo 2007; Valverde \& Näslund-Hadley, 2010), en líneas generales los estudiantes no son competentes en esta disciplina. En el Perú, distintas evaluaciones (Morales \& Frisancho, 2013; PISA, 2012; SICRECE 2015; UMC, 2013, 2014, 2015; UNESCO 2014; Valverde et al., 2010) dan cuenta de su poca competencia matemática, tanto en la educación básica como en la superior. La Evaluación Censal de Estudiantes (ECE - UMC 2013, 2014, 2015) realizada en segundo grado de primaria, muestra estas dificultades. En las evaluaciones de los años 2013, 2014 y 2015 solo el $16.8 \%, 25.9 \%$ y $26.6 \%$ de los estudiantes, respectivamente, lograron los aprendizajes esperados para su grado, mientras que la mayoría logró aprendizajes parciales o pudo realizar solamente tareas de poca exigencia.

En la región Ucayali de la Amazonía del Perú los resultados son aún más bajos, pues aunque en el 2015, siguiendo la tendencia nacional, se observa una mejoría con respecto a los años 2013 y 2014, solo el $10.3 \%$ de estudiantes alcanza el nivel satisfactorio en ese año, siendo Ucayali una de las regiones con los peores resultados nacionales en esos tres años. En esta región, el 79.4\% de estudiantes del sector rural alcanza el nivel en inicio en el año 2015, versus el $49.8 \%$ de estudiantes urbanos; de estos últimos, el $11.4 \%$ alcanza el nivel satisfactorio, mientras que solamente el 2.3\% logra este nivel en el sector rural. Si bien no se ha evaluado la competencia matemática en niños de escuelas interculturales bilingües
(EIB), sí existen experiencias de evaluación de matemática en castellano, en segundo grado, en las Provincias de Atalaya, Coronel Portillo, Padre Abad y Purús, todas de la región Ucayali, en las que hay población indígena (SICRECE 2015). La mayoría de los estudiantes se encuentra en el nivel más bajo de la competencia matemática, lo que indica que no han desarrollado las capacidades matemáticas esperadas para su edad.

Diversos factores pueden explicar estos resultados, por ejemplo, el poco conocimiento de los profesores con respecto a la Matemática y su enseñanza, o sus creencias sobre su naturaleza epistemológica y los apoyos que son necesarios para producir aprendizajes significativos en esta área (Becker 2001, 2012; Gascón, 2001; Moreano, Asmad, Crus \& Cuglievan, 2008; Otero, Vizcaino \& Carmenates, 2015; Teixeira, Abud, Ribeiro \& Fernandes, 2015). Otro factor es la existencia de planes curriculares y materiales de aprendizaje poco eficaces, desvinculados de los saberes culturales de los niños y de lo que realmente ocurre en las aulas, donde priman la memorización, la mecanización, la selección de unos temas en desmedro de otros y la poca o a veces equivocada retroalimentación de parte de los profesores hacia los estudiantes (Cueto, Ramírez, León \& Pain, 2003; Da Costa \& de Lucena, 2015; Ministerio de Educación, 2013, 2009; Valverde \& Näslund-Hadley, 2010). Una razón adicional es que los programas escolares suelen no estar guiados por el desarrollo psicológico, y plantean metas educativas muy poco oportunas (Kamii, 2000; Piaget 1978). Por ejemplo, en el Diseño Curricular Nacional (MINEDU, 2009) la sustracción aparece como meta del primer grado de nivel primario y en la Propuesta de Diseño Curricular con Enfoque Ambiental (MINEDU, 2011), documento de la Dirección Regional de Educación de Ucayali (DREU) para las escuelas de la región, si bien la sustracción no se plantea para el primer grado, en segundo grado se pide que los estudiantes la trabajen con números de hasta tres cifras, lo 
que resulta inadecuado ya que la sustracción exige tener claras las nociones referentes a la relación parte-todo (Piaget, 1977b) y la capacidad de pensar en negativo (Piaget, 1978), las que no se encuentran desarrolladas en niños menores de 7 años. El Diseño Curricular Nacional del año 2016 no corrige estas metas.

Se sabe también que la variable socioeconómica se asocia con bajos resultados en Matemática y con las expectativas que tiene el estudiante sobre su propio aprendizaje, siendo los de menores recursos los que manifiestan expectativas más bajas sobre sus aprendizajes y obtienen los peores resultados (Barrenechea, 2010; Bos, Ganimian \& Vegas, 2013; Carnevale, 2005; Cueto, Guerrero, León, Zapata \& Freire, 2013; Ravela, 2002; Treviño, 2006). Sin embargo, es importante observar que las evaluaciones estandarizadas pueden no ser adecuadas para estudiantes indígenas (Nelson-Barber \& Trumbull, 2007; Treviño, 2006) al no tomar en cuenta sus conocimientos particulares ni sus modos de pensar. Diversas investigaciones plantean que el aprendizaje de la Matemática tiene mejores resultados cuando está contextualizado culturalmente (Adams, Luitel, Afonso \& Taylor, 2008; Dewah \& Van Wyk, 2014; Fyhn, Jannok Nutti, Nystad, Eira \& Hætta, 2016; Nunes, 1993; Nunes \& Bryant, 1996; Nunes, Carraher \& Dias Schliemann, 1982; Tillema, 2012) ya que, si bien la Matemática es un conocimiento de naturaleza universal (Piaget, 1975), el contexto sociocultural en el que se desarrolla y las actividades tradicionales y prácticas matemáticas cotidianas con las que el niño está familiarizado, son importantes e influyen en su aprendizaje. Las matemáticas no se construyen por repetición sino por medio de las relaciones que el propio niño crea a partir de su interacción con los objetos (Flavell, 1976; Kamii, 1985, 1994, 2000; Piaget, 1971; Piaget \& Inhelder, 1980). En este sentido, evaluaciones de la capacidad matemática que incluyan situaciones y actividades culturalmente pertinentes identificarían mejor el verdadero potencial de los niños y deberían usarse para evaluarlos.

A pesar de la importancia del conocimiento lógico-matemático, este casi no ha sido evaluado en la Amazonía Peruana. Las evaluaciones a gran escala que realiza el Ministerio de Educación permiten abarcar muestras grandes, pero no profundizan en los procedimientos que los niños utilizan para resolver los problemas. Por su propia naturaleza, este tipo de evaluación se enfoca en las respuestas finales, desatiende los procesos y no recoge los procedimientos que ha construido el niño. Como se ha visto, este tipo de evaluación podría no capturar por completo el conocimiento de los estudiantes indígenas, además de contribuir al aprendizaje memorístico e influir en la forma de enseñar de los docentes y en lo que los grupos aprenden al hacer que los profesores entrenen a los estudiantes para afrontar las pruebas y no los problemas de la realidad (Monereo, 2014; Woolfolck, 2010; Zavala, 2012).

El estudio que se informa, realizado con niños de la comunidad del pueblo Shipibo konibo, se suma a la escasa investigación constructivista en la academia peruana (Meza \& Sirlopú, 1997) y a las investigaciones que han estudiado el desarrollo del pensamiento desde la perspectiva piagetiana en diversos contextos interculturales (Adjei, 1977; Bovet, 1974; Bruner, 1966; Dasen, 1984; Maynard, 2008; Mishra, 2014; Opper, 1977; Price-Williams, 1961), compara el rendimiento en Matemática de un grupo de niños de una comunidad Shipibokonibo de la región Ucayali del Perú, utilizando dos tipos de evaluación, una tradicional de lápiz y papel basada en el Diseño Curricular Nacional (MINEDU, 2009), en las Rutas de aprendizaje (Ministerio de Educación, 2015) y la Evaluación Censal de Estudiantes (ECE) y otra que es la Entrevista Clínico-crítica de Jean Piaget (Delval, 2001; Ducret, 2014; Parrat, 2016a) e incluye problemas contextualizados y material concreto manipulable o representativo.

El pueblo Shipibo - konibo es el producto de una combinación cultural de tres grupos distintos, los shipibos, los konibos y 
los shetebos. Este pueblo se encontraba tradicionalmente asentado en la ribera del río Ucayali y sus afluentes, aunque debido a la migración, actualmente se ubica en las regiones Ucayali, Madre de Dios, Loreto y Huánuco, y en la ciudad de Lima. En el año 2007 la población shipibo - konibo llegaba a los 22.517 habitantes (Ministerio de Cultura, 2014). Los niños están familiarizados con el dinero, pues cuentan con una pequeña bodega que les ofrece oportunidades para hacer cálculos en actividades de compra y venta. Muchas madres de familia se dedican a la artesanía, ya sea el bordado tradicional Kené (ver Belaunde, 2012; Brabec \& Mori 2009), o a la producción de bisutería a base de semillas. Ambas cosas se comercializan. Los padres de familia realizan transacciones comerciales en la comunidad y en la ciudad de Pucallpa y llevan muchas veces con ellos a sus hijos. Los niños, además, tienen muchas oportunidades de recolectar y manipular objetos (semillas, palos de madera, etc.), contarlos, agruparlos, romperlos o partirlos por la mitad.

\section{Método}

\section{Participantes}

Participaron 5 niñas y 2 niños de 7,9 y 11 años que cursaban la enseñanza primaria en una escuela bilingüe multigrado de una comunidad Shipibo-konibo de la región Ucayali, en la Amazonía del Perú. Tres niños estaban en segundo grado, dos en tercero, uno en quinto y uno en sexto. Su lengua materna es el shipibo, aunque todos dominaban el español. La selección de los niños fue aleatoria.

\section{Procedimientos e Instrumentos}

La evaluación se llevó a cabo en un aula de la escuela. La duración fue variable, siendo más breve con los estudiantes de 11 años (30 minutos) y mayor con los de 7 años (45 minutos). Las tareas se presentaron siempre en el mismo orden: primero las entrevistas (adición y sustracción) y luego la evaluación de lápiz y papel.

Se contó con el consentimiento de la comunidad, incluyendo al jefe de la misma, el profesor responsable de la escuela y los padres de familia. Se utlizó el protocolo de consentimiento informado de Frisancho, Delgado y Lam (2015), que surge de investigaciones previas con comunidades indígenas amazónicas e incluyó una asamblea comunitaria de consentimiento y una devolución de resultados. A los niños se les explicó el objetivo de la evaluación y se les pidió su asentimiento. La evaluación contó con dos investigadores y se realizó en español.

Se administraron la Entrevista ClínicoCrítica con material concreto representativo o manipulable y la Prueba de Lápiz y Papel.

La Entrevista Clínico-Crítica indaga en la lógica y el conocimiento del niño (Ducret, 2004; Parrat, 2016a) y permite al evaluador formular contra-ejemplos para profundizar en su razonamiento. Se interesa en los niveles sucesivos de organización de la conducta y valora los cambios cualitativos por sobre los cuantitativos, es decir, desea observar la lógica y los procedimientos mentales y no únicamente las respuestas finales de los niños para la resolución de problemas. La Entrevista Clínico-Critica permite llegar a conclusiones no solo en torno a lo que el evaluado manifiesta verbalmente, sino también a partir de la observación de la forma en que manipula los materiales (Delval, 2001). El evaluador puede ir orientando al niño que no muestra alguna estrategia para resolver el problema, haciéndole preguntas o sugiriéndole procedimientos, siguiendo una variante de la Entrevista Clínico-Crítica propuesta por Inhelder (Parrat, 2016a, 2016b).

Para hacer la evaluación con el método clínico-crítico se plantearon problemas aritméticos de adición y sustracción, contextualizados en actividades culturales cotidianas con la que los niños estaban familiarizados. Se incluyó material representativo y material manipulable que podían utilizar para resolver los problemas. El 
material representativo consistió en figuras de animales u objetos, mientras que el manipulable fueron bolitas blancas y negras e hilo de pescar para armar collares. En los Cuadros 1 y 2 se presentan los contextos en los que se sitúan los problemas, el problema en sí y las preguntas.

La Prueba de Lápiz y Papel consta de 12 ejercicios, 6 de adición y 6 de sustracción (ver Cuadro 3). Para generar mayor contraste con la evaluación anterior y evitar la posible interferencia de la comprensión lectora, se construyeron ítems sin contexto ni planteo de problema, siguiendo el formato incluido en las evaluaciones nacionales (UMC, 2013, 2014, 2015).

El Cuadro 4 muestra los niveles de desempeño que pueden alcanzarse tanto en la evaluación con la Entrevista Clínico-Crítica como en la prueba escrita.

\section{Resultados}

\section{Operación de adición mediante la En- trevista Clínico-Crítica}

Se observa una primera etapa de no-composición aditiva, una etapa intermedia de composición intuitiva y finalmente, una etapa de composición aditiva lograda en la que el niño tiene pensamiento reversible y entiende la propiedad conmutativa, así como que el resultado de la suma se mantiene, aunque los sumandos se organicen de distinto modo. Estas etapas van de la mano con las identificadas por Piaget (1971).

\section{tiva.}

Etapa inicial de no-composición adi-

A los 7 años no existe composición aditiva. Los niños son incapaces de unir dos cantidades distintas para construir una nueva. Además, presentan errores al momento de contar y en algunos casos dan respuestas distintas, ilógicas y contradictorias entre sí. Por ejemplo:

Wil (7 años): Supongamos que tú y yo salimos a cazar y hoy cazamos 4 perdices $y$ mañana cazamos 5. ¿Cuántas perdices cazamos en total? - Cuatro -Hoy cazamos 4 perdices y mañana cazamos 5, ¿cuántas perdices cazamos en total? -Once.

Debido a que no posee la capacidad de iterar (unir dos cantidades para formar una nueva), da respuestas al azar sin una lógica que las sustente. A continuación se le propone hacer uso del material para resolver el problema:

- Si deseas puedes usar las figuras para contar, por ejemplo - (El niño pone una a una las figuras contando del uno al quince) -Entonces decíamos que hoy miércoles cazamos 4 perdices y mañana jueves cazamos 5 , ¿cuántas perdices cazamos en total? - Seis.

Wil no parece recordar las dos cantidades que debe adicionar y realiza un conteo que no toma en cuenta esos números. Cuando se le repregunta, vuelve a dar respuestas carentes de lógica. Debido a esto, se intenta otra estrategia para ayudarlo:

-A ver, dame 4 perdices - (El niño pone 4 figuras en la mano izquierda del evaluador) -Muy bien, ahora dame las 5 que cazamos al dia siguiente - (pone 5 figuras en la mano derecha del evaluador) - Si yo uniera las perdices de esta mano (mano izquierda) con la de esta mano (mano derecha), ¿cuántas perdices tendría en total? Diez - A ver vamos a comprobarlo (el evaluador pone todas las figuras en hilera y le pide que las cuente) - (El niño cuenta 9 figuritas) - ¿Entonces cuántas perdices hay en total? - (El niño ríe) - Por ejemplo, si en un día cazamos estas (4 figuras) y otro día estas de acá (5 figuras) ¿cuántas cazamos en total? - Jueves - Por ejemplo, si yo junto estas de acá (4 figuras) con estas de acá (5 figuras), ¿cuántas tendría en total? - Miércoles. - ¿Tendría una perdiz? - (Asiente con la cabeza) - ¿Tendría dos perdices? (Asiente con la cabeza).

\section{tiva.}

\section{Etapa de composición aditiva intui-}

Los niños en esta etapa tienen un conocimiento más desarrollado del concepto de 
suma y además, menos errores en el conteo. Hay intentos de resolver el problema por medio de algoritmos mentales; sin embargo, cuando hacen uso de este recurso suelen cometer errores. Los niños ya tienen claro que para llegar a la respuesta deben iterar los elementos, pero tienen dificultades para trabajar con cantidades grandes y en ocasiones, suelen cambiar su respuesta ante las repreguntas. Por ejemplo, se le propone a Man una situación en la que debe realizar la operación $4+5$ :

Man (9 años): - Vamos a suponer que el lunes yo cacé 4 perdices y el martes cacé 5 perdices, ¿cuántas perdices tengo en total? - (pone en hilera 4 tarjetas, luego pone una más y dice súbitamente que el resultado es 9). ¿Cómo has llegado a esta respuesta? He juntado.

Man itera naturalmente los elementos y no necesita hacer un uso completo del material para llegar a la respuesta. Sin embargo, su desempeño cambia cuando se le pide resolver un problema que implica trabajar con números de mayor tamaño:

Man (9 años): Ahora resulta que tú y yo salimos a cazar y logramos cazar 21 perdices, pero cuando estamos regresando nos encontramos con un vecino y nos regala 15 perdices más, ¿cuántas perdices tenemos ahora en total? - (pone 21 perdices en hilera sobre la mesa contando una por una, luego pone 15 perdices también en hilera contando una por una. Finalmente cuenta todas una por una, pero omite un número en su conteo) - Treinta y cinco.

A diferencia de la situación anterior, no puede llegar a la respuesta mentalmente y necesita usar el material. Además, el recurso de conteo no se encuentra consolidado, pues Man tiende a fallar al momento de trabajar con números de dos cifras. En esta etapa, el uso de algoritmos induce a error, tal como se observa en el caso de Les:

Les (9 años): Supongamos que un papá y un hijo salieron juntos a cazar perdices $y$ en un momento se separaron, el papá se fue por un lado y el hijo por otro lado. Resulta que el papá, como es más hábil, cazó
13 perdices y el hijo cazó 5. Cuando llegan a su casa juntan todas las perdices, ¿cuántas perdices tienen en total? - Ocho - ¿Y cómo lo sabes? - Así, todo he juntado. - Si juntas las 13 más las 5 te dan 8, ¿quieres mostrarnos con las tarjetitas? Yo te contaba que el papá cazó 13 y el hijo cazó 5 - ¿Trece di? - Sí, trece - (El evaluado va poniendo una a una en un montículo hasta llegar a 13 perdices. Luego en otro montículo pone $5 \mathrm{fi}$ guras. Finalmente cuenta los dos montículos de corrido) Son 19.

Les no logra llegar a la respuesta correcta porque hace un mal uso del algoritmo. Se puede deducir de su primera respuesta $(13+5=8)$ que solo sumó las unidades de los dos números. Cuando hace uso del material para llegar a la respuesta presenta un error de conteo.

Finalmente, otra característica de esta etapa es la ausencia o poco desarrollo del pensamiento reversible. Por ejemplo, cuando se presenta la operación 8+9 Man llega a la respuesta, pero cuando se plantea la operación inversa $(9+8)$, no deduce que el resultado es el mismo y realiza todo el procedimiento de nuevo.

Man (9 años): Ahora me gustaría que me ayudes a hacer unos collares, ¿te parece? Si-Supongamos que tengo un collar que tiene 8 bolitas y le quiero agregar 9 bolitas más ¿cuántas bolitas tendría el collar? (El niño intenta mentalmente) - ¿No te gustaría armar el collar para saber la respuesta? - (pone 8 bolitas en el hilo, de una en una) - Ahora a ese collar le queríamos agregar 9 bolitas más ¿no? - (agrega 9 bolitas de una en una) - ¿cuántas bolitas tiene ahora en total el collar? - (cuenta todas las bolitas de una en una y logra saber que el resultado es 17). Supongamos ahora que tengo un collar que tiene 9 bolitas y quiero agregarle 8 bolitas más ¿cuántas bolitas tendría el collar en total? - (El niño retira todas las bolitas del collar y realiza el procedimiento nuevamente, esta vez empezando por el número 9) Son diecisiete. 


\section{Etapa de composición aditiva lograda.}

Los niños que se encuentran en esta etapa han consolidado el concepto de suma y no tienen dificultades para operar mentalmente con números pequeños. Tratan de llegar a la respuesta mentalmente y presentan resistencias al momento de hacer uso del material. Conciben como estrategia natural el conteo para llegar a la respuesta y comprenden que cambiar el orden de los factores en una adición no altera el resultado de la misma. Sin embargo, si bien logran operar mentalmente con números pequeños, cuando estos son más grandes pueden equivocarse, aunque sus errores son más lógicos que en la etapa anterior. Por ejemplo, se le plantea una operación a Sha $(15+18)$ y se le propone hacer uso del material para llegar a la respuesta, pero intenta resolver el problema mentalmente:

Sha (11 años): Ahora, si tenemos un collar que tiene 15 bolitas blancas y luego le agregamos 18 bolitas negras, ¿cuántas bolitas tendríamos en total? - (El niño permanece en silencio intentado sumar mentalmente) - ¿Deseas armar el collar? - Sí (empieza agregando una por una las 18 bolitas negras) - ¿cuántas bolitas tiene el collar? - Dieciocho - Perfecto, ahora tenemos que agregarle 15 blancas. A ver aqui tengo 5 bolitas blancas, ¿si le agrego 5 más cuántas son? - Diez - ¿Y si le agrego 5 más cuántas son? - Quince - Muy bien, ahora tenemos estas 15 bolitas blancas y estas 18 bolitas negras, si juntamos todo ¿cuántas bolitas hay en total? - (Intenta llegar a la respuesta mentalmente) - ¿cómo harías para saber cuántas bolitas tendría el collar? - (permanece en silencio).

A pesar de que Sha tiene a su disposición el material manipulable, intenta llegar al resultado sumando mentalmente. En vista de esto, el evaluador lo induce a utilizarlo:

- Supongamos que tú tienes 15 bolitas blancas y te encuentras con una amiga que tiene 18 bolitas negras. Para poder hacer un collar más grande juntan todas las bolitas. Luego ella te pregunta, ¿y ahora cuántas bolitas tiene el collar? ¿Tú qué le di- rías? - Treintaiuno - ¿Y cómo supiste eso? - (El niño permanece en silencio) - ¿cómo harías para revisar y estar segura? - Contar - A ver, muéstranos cómo contarías Aqui hay 18 (refiriéndose a las negras) entonces 19, 20, 21, 22, 23, 24, 25, 26, 27, 28, 29, 30, 31, 32, 33 (refiriéndose a las blancas) - Muy bien, entonces primero tu contaste las 18 y luego empezaste con las blancas y dijiste 19, 20, 21... ¿Y si lo hicieras al revés? Si contaras primero las 15 y luego las 18, ¿daría el mismo resultado? - Sí ¿no importa que esté al revés? -No.

La primera respuesta, Sha (31) la obtuvo sumando mentalmente. Luego, al revisarla por medio del conteo, llegó a la respuesta correcta. Esto muestra que los estudiantes que se encuentran en la etapa más avanzada de la operación de adición, todavía pueden tener dificultades para sumar mentalmente dos números de dos dígitos. Sin embargo, en esta etapa los niños no solo suelen cometer menos errores sino que sus respuestas incorrectas son más lógicas. Por ejemplo, afirmar que trece más ocho es igual a cinco $(13+8=5)$ es sin duda menos lógico que decir que quince más dieciocho es igual a treinta y tres $(15+18=33)$, ya que es imposible que se obtenga como respuesta un número de un dígito (menor a la decena) a partir de la suma de dos números, uno de los cuales es mayor que 10 . Finalmente, es importante resaltar que, como se expresa en el caso de Sha, los estudiantes de esta etapa presentan un pensamiento reversible que les permite entender la propiedad conmutativa de la adición.

\section{Operación de adición en la prueba de lápiz y papel.}

Los niños de 7 años dejaron la Prueba de Lápiz y Papel en blanco. Los de 9 años sí presentan resoluciones. La Figura 1 muestra como Man llega a la respuesta usando palotes.

El recurso que utiliza Man es deficiente, pues no tiene ningún orden para llegar a la respuesta. Por ejemplo, no agrupa los palotes para facilitar el conteo y evitar errores. 
Además, el procedimiento demanda demasiado tiempo, pues implica representar cada uno de los números y luego contar la totalidad. En la Figura 2 se observa otro tipo de resolución, realizada por Les, de 9 años.

Se observan intentos por resolver la operación por medio del procedimiento algorítmico, posicionando un número arriba del otro para sumar primero las unidades y luego las decenas. No obstante, a pesar de que pone los números en la posición correcta, no efectúa bien los pasos y no obtiene la respuesta correcta. Sin embargo, en la prueba basada en tareas Les obtiene respuestas más lógicas; por ejemplo, en la suma $21+15$ afirma que la respuesta es 37 , el cual es un número más cercano a la respuesta correcta y en el caso de la suma $8+9$ obtiene la respuesta correcta.

Los estudiantes de 11 años pueden escribir el resultado de forma directa, simplemente sumando mentalmente, o hacer uso del procedimiento algorítmico. En la Figura 3 se muestran resoluciones de Dal que son un ejemplo de lo segundo.

\section{Operación de sustracción mediante la Entrevista Clínico-Crítica.}

\section{Ausencia de sustracción.}

Por lo general, los niños de 7 años no emplean una estrategia para realizar la sustracción, y dan respuestas diferentes a cada pregunta:

Wil (7 años): Supongamos que salimos a cazar y llevamos catorce flechas, ipuedes poner catorce flechas? - (Pone una a una las 14 flechas) - De esas catorce flechas usamos 6 , ¿con cuántas flechas regresamos a casa? - Siete - Si tenemos catorce flechas y perdemos 6 en el bosque, ¿con cuántas regresamos a casa? - Seis. - A ver, ¿puedes darme las 6 flechas que utilizamos? - (El niño pone en la mano del evaluador 6 flechas, una a una y contando en voz alta) - ¿con cuántas flechas regresamos a casa? - (Cuenta las flechas que quedan) Ocho.

En este ejemplo se observa que el niño no logra la respuesta por sí solo y necesita ayuda para entender que el procedimiento correcto es retirar 6 flechas del grupo de 14. En el caso de Luc, el evaluador lo guía para que utilice el material y pueda efectuar la sustracción:

Luc (7 años): Vamos a suponer que en casa tenemos 18 flechas, ¿puedes poner las 18 flechas? - (Pone una a una y en hilera las 16 flechas, luego cuenta todas para verificar cuántas tiene, seguidamente agrega dos más) - Bien, acá tenemos 18 flechas. Ahora supongamos que tu papá se lleva 12 flechas de estas 18, ¿con cuántas flechas nos quedamos en casa? - (Cuenta 12 flechas y las separa de las restantes, luego cuenta una a una las restantes) Seis.

Sin embargo, cuando se le pide una explicación sobre su acción, no la logra. Luego, al preguntarle sobre la acción contraria a la que ha realizado (juntar nuevamente las 6 flechas con las 12 flechas), afirma que al juntar de nuevo las dos cantidades habría menos que antes.

- ¿cuáles son las flechas que se quedaron en casa? - (El niño señala el grupo de 6 flechas) - ¿Y cuáles son las que se llevó el papá? - (Señala el grupo de 12 flechas) - ¿Si juntamos las que quedan con las que se llevaron tenemos menos, más o igual que las que habian antes? - Menos. - ¿qué faltaría para tener igual que antes? - (Permanece en silencio).

En este nivel los niños no pueden realizar sin ayuda la operación de sustracción. Además, cuando han sustraído no reconocen que al juntar nuevamente las cantidades resultará el mismo número, lo que indica que la resta y la suma no son conceptualizadas como recíprocas.

\section{Sustracción en proceso.}

Los niños de 9 años tienen mayor claridad con respecto a la estrategia para resolver el problema y comprenden que se debe extraer una cantidad de una totalidad mayor. Aunque tienen éxito en sus respuestas y reconocen los números con los que han operado, no logran explicar sus acciones, y a veces no son conscientes de que la suma y la resta son operaciones complementarias: 
Les (9 años): Supongamos que queremos ir a cazar animales y para esto tenemos en casa 8 flechas - (Pone 8 flechas, una por una, sobre la mesa) - Pero, resulta que cuando vamos a buscar esas 8 flechas descubrimos que alguien se ha llevado 3, ¿cuántas flechas hemos encontrado? - (Separa 3 flechas de las 8 flechas que puso inicialmente sobre la mesa, luego cuenta las flechas que le quedan después de haber quitado 3) Cinco - ¿Cinco, no? ¿Cómo lo sabes? ¿Qué es lo que has hecho para saber que deberíamos encontrar 5? - (El niño permanece en silencio) - ¿Y cuáles son las que le quedan? - (Señala el grupo de las 5 flechas) - ¿Y cuáles son las que se han llevado? - (Señala el grupo de las 3 flechas). - Ahora, una pregunta, si es que nos devuelven las tres flechas que se llevaron ¿cuántas flechas tendríamos? - (Permanece en silencio)

\section{Sustracción lograda.}

Los estudiantes son capaces de llegar mentalmente a la respuesta correcta de una resta cuando esta implica operar con dos números de una cifra. Además, cuentan con pensamiento reversible:

Sha (11 años): Ahora supongamos que vamos a ir a cazar, asi que tenemos que ir a buscar nuestras flechas. Supongamos que teníamos 8 flechas, pero tu papá se llevó 3 flechas, ¿cuántas flechas tenemos? - Cinco. - ¿nos podrías mostrar con las tarjetas? - (El niño pone 8 flechas sobre la mesa en hilera) - Si tu papá se lleva 3 flechas, ¿cuántas flechas nos quedarían? - Cinco - Y si juntamos nuevamente las que se llevó tu papá con las que se quedan, ¿tendríamos la misma cantidad de antes? - Sí.

Cuando se enfrentan a números de dos dígitos, ya no les es posible realizar el cálculo mental y se ven obligados a utilizar las tarjetas de apoyo. En estos casos se puede observar también un pensamiento reversible. Por ejemplo:

Dal (11 años): Supongamos que tienes en casa 22 tapetes, pero la vecina te pide 14 tapetes prestados, ¿cuántos tapetes te quedan en casa? - (Pone una a una 22 tarjetas) - Le prestamos 14 a la vecina - Sacamos 14 (Cuenta 14 tapetes y los retira) - ¿cuántos tenemos en casa? - (Cuenta los restantes uno por uno) Ocho - Y por ejemplo, ¿si juntamos esto (los 14 tapetes) con estos (los 8 tapetes) tendremos lo mismo que antes? - Sí, lo mismo - Un vez le pregunté esto a una niña de tu edad y me dijo que habría una cantidad diferente. - No - ¿cómo nos mostrarías que está equivocada? - Lo junto así y cuento (Junta todos los tapetes).

El niño necesita el material representativo para resolver el problema, pero su manipulación de dicho material y sus explicaciones demuestran que ha comprendido plenamente la operación que realiza.

\section{Operación de sustracción con la prueba de lápiz y papel.}

Los niños tienen un mejor desempeño en la evaluación basada en tareas que en la Prueba de Lápiz y Papel. Los de 7 años no resolvieron esta prueba. A los 9 años ya muestran algunos intentos de resolución, aunque muchas veces fallidos (ver Figura 4).

Pretenden llegar a la respuesta haciendo uso de palotes, pero estos se adicionan, no se sustraen. Esto puede deberse a que no reconoce el símbolo de sustracción. En la Figura 5 se muestra el intento de Les de usar un método algorítmico.

En este caso, el niño escribe el símbolo de sustracción como parte de su procedimiento, pero pareciera querer adicionar los números, aunque tampoco obtiene el resultado correspondiente a una adición. Finalmente, en la Figura 6 se pueden observar dos ejemplos del uso de algoritmos realizados por Dal, de 11 años (Figura 6).

El estudiante no domina el procedimiento algorítmico. En el primer caso (16-9), por ejemplo, entiende que la respuesta es 7, pero olvida que ya no debe bajar el uno que representa a las decenas del número 16. El uso de algoritmos, que aparentemente se ha mecanizado sin haber sido plenamente comprendido, lo lleva a una respuesta ilógica. En la evaluación basada en tareas, este niño sí obtiene las respuestas correctas. 
Para comparar los resultados obtenidos por los niños en ambos tipos de prueba, se presentan las Tablas 1 y 2 .

Como puede verse, ningún estudiante de 7 años resuelve la prueba escrita ni de adición ni de sustracción. Sin embargo, en la entrevista, aun cuando fallan en alcanzar el resultado correcto, evidencian un procedimiento, lo que significa que los problemas cotidianos con argumentos los hacen razonar. Los de 9 años muestran más variación en sus resultados de la adición, pero fallan en la prueba escrita de sustracción, logrando mejores resultados en esta operación en la Entrevista Clínica Crítica. En el caso de los niños de 11 años, es casi indiferente cuál método usan, aunque los ejercicios tanto de suma como de resta corresponden a los 7 años según el currículo oficial y podrían haber resultado muy sencillos para ellos. En todos los casos, con el método clínico crítico se puede observar el razonamiento de los niños, los procedimientos que utilizan, así como sus dificultades al resolver los problemas (por ejemplo, errores de conteo), lo que no es posible apreciar con la evaluación escrita.

\section{Discusión}

Se investigó el desempeño en las operaciones de adición y sustracción de siete estudiantes de primaria de una comunidad Shipibo-konibo, los que fueron evaluados con el método clínico - crítico de Jean Piaget (Delval, 2001; Ducret, 2004; Parrat, 2016a) y también mediante una prueba tradicional de lápiz y papel. Los niveles encontrados en la evaluación de la adición, con el método clínico - crítico, responden al proceso de desarrollo del pensamiento aditivo que se discute en La génesis del número (Piaget, 1971), donde se plantea una primera etapa que se caracteriza por la imposibilidad de iterar (adicionar) elementos, una segunda en la cual los niños pueden iterar pero cambian sus respuestas frente a contra sugestiones, y una tercera en la que la operación se encuentra consolidada. En este estudio, los estudiantes de 7 años se ubican en el primer nivel, los de 9 años en el segundo y los de 11 en el tercero. Los niveles encontrados en la evaluación de la operación de sustracción mediante el método clínico-crítico (Nivel 1: Ausencia de la sustracción. Nivel 2: Sustracción en proceso. Nivel 3: Sustracción lograda) describen el desarrollo de la capacidad para pensar en negativo y de la reversibilidad del pensamiento, y responden también a la teoría piagetiana (Piaget, 1978). En este sentido, esta investigación puede considerarse una evidencia más de la universalidad del conocimiento lógicomatemático (Piaget, 1972). Más allá de la naturaleza cultural de los elementos que se sumen o resten (sean manzanas, semillas, carritos de juguete o flechas), las operaciones que se realizan son universales y la secuencia de desarrollo de estas operaciones también lo es (Piaget, 1971), pues independientemente de la cultura a la que pertenece, todo ser humano cuenta con las mismas estructuras lógico-matemáticas para entender y organizar el mundo (Hallpike, 1986; Piaget \& García, 1982).

Aunque los participantes de este estudio presentan niveles de desarrollo de las operaciones de adición y sustracción similares a los encontrados por Piaget y sus seguidores en sus estudios originales, es importante notar que estos se están logrando tardíamente. La teoría Piagetiana indica edades aproximadas para el paso de un estadio al otro; sin embargo, la velocidad con la que este conocimiento se desarrolla depende, en buena medida, de las características y exigencias del contexto (Dongo, 2002; Hallpike, 1986; Piaget, 1977a). Los niños de comunidades indígenas suelen desarrollar capacidades distintas a las de un niño occidental y urbano, por ejemplo, mucha mayor capacidad de observación y de trabajar colaborativamente (Chavajay \& Rogoff, 2002; Paradise, 1991, 1996; Paradise \& Rogoff, 2009; Rogoff, 1993, 2003). Sin embargo, estos mismos niños pueden tener dificultades en tareas escolares con las que se encuentran menos familiarizados (Dongo, 2002, 2009, 2011; Lacasa, 1989; Rogoff, 1993). Por ejemplo, algunos estudios muestran que el grado de escolaridad de los padres influye en el desarrollo cognitivo 
de los niños (Ignat \& Nataly, 2015; Lipina, Martelli, Vuelta, Injoque-Ricle \& Colombo, 2004; Musso, 2010; Pascual, 2008; Rindermann \& Baumeister, 2015), y existe evidencia de que los niños en situación de marginación, opresión y pobreza suelen tener un desarrollo cognitivo más lento que los que crecen en contextos económicamente privilegiados (Dicker- son \& Popli, 2016; Dongo, 2002; Lacunza, Contini \& Castro, 2010). Esto ayuda a entender que los participantes de este estudio hayan mostrado niveles de desarrollo inferiores a los esperados para su edad, al igual que los viene mostrando la región Ucayali, en las evaluaciones censales (UMC, 2013, 2014, 2015) sobre todo en el área rural. Sin embargo, tal como muestra este estudio, una evaluación culturalmente contextualizada, cualitativa y administrada individualmente, permite una mejor aproximación a las capacidades de los niños y brinda información acerca de su nivel de desarrollo en las operaciones evaluadas y su forma de afrontar los problemas. Contar con los dedos, con partes del cuerpo, o con otro tipo de ayuda, es decir, realizar la acción en un plano material (Galperin, 1969), es una conquista cultural importante que funciona como un andamio para pasar de lo que Vygotsky (1983) llama la Aritmética natural, a la Aritmética cultural formalizada. En este sentido, la investigación realizada permite también dar luz sobre la génesis de los procesos de conteo, identificando las estrategias que utilizan los niños en distintos momentos del desarrollo. Resultaría entonces pertinente utilizar el método clínico crítico en la investigación, con la finalidad de identificar cualitativamente los niveles de logro de los estudiantes y explicar los mecanismos del proceso de resolución de problemas y los niveles de pensamiento matemático que han desarrollado. Esto brindará información al docente para comprender mejor los procesos de construcción de las operaciones aritméticas, tomar mejores decisiones en las sesiones de aprendizaje, ajustar mejor su práctica pedagógica y potenciar aprendizajes en contextos culturalmente diversos

Para futuros estudios se recomienda identificar las trayectorias educativas de los niños, de modo que se sepa, por ejemplo, si han asistido o no a la educación inicial, ya que esta es importante como base para el desempeño escolar posterior (Aunio \& Niemivirta, 2010; Cueto \& Díaz, 1999; Wolfgang, Stannard \& Jones, 2001). Igualmente, se sugiere contar con mayor información de las familias, por ejemplo, el grado de escolaridad de los padres, a fin de identificar su relación con los resultados obtenidos por los niños. Se recomienda también, para estudios posteriores, ampliar la muestra y centrarse en una misma edad o grado escolar, para de este modo obtener información más precisa sobre dicho grupo y proponer estudios futuros para otras edades. Finalmente, resultaría importante incluir a los docentes en estudios posteriores sobre este tema, de modo que se puedan conocer sus estrategias pedagógicas y su nivel de preparación para la enseñanza de la matemática. 


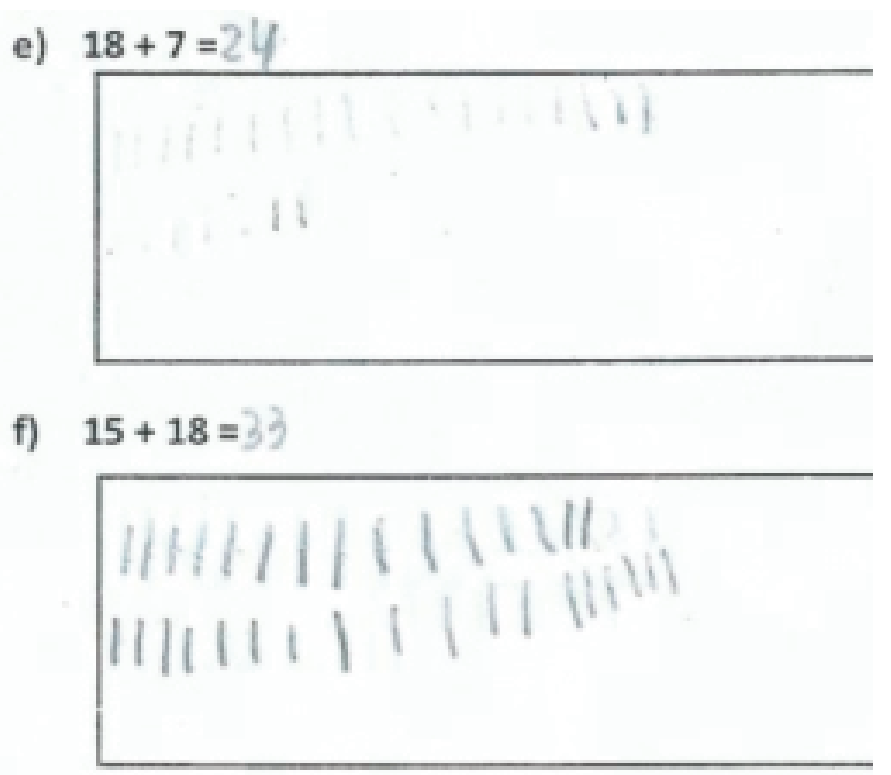

Figura 1. Resolución de operación de adición (Man, 9 años)

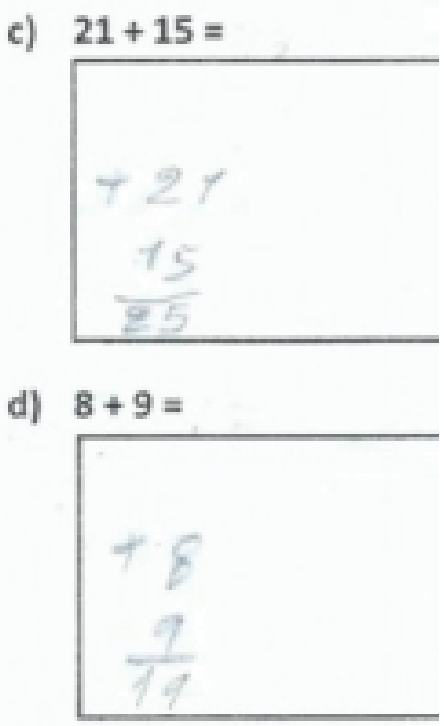

Figura 2. Resolución de operación de adición (Les, 9 años) d) $8+9=17$

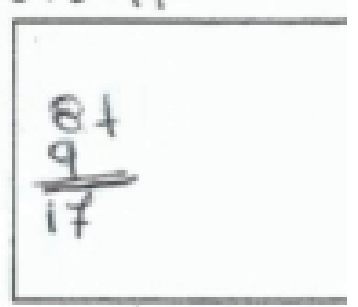

e) $18+7=25$

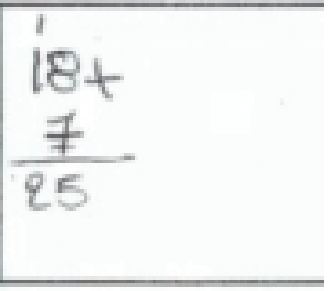

Figura 3. Resolución de operación de adición (Dal, 11 años) 


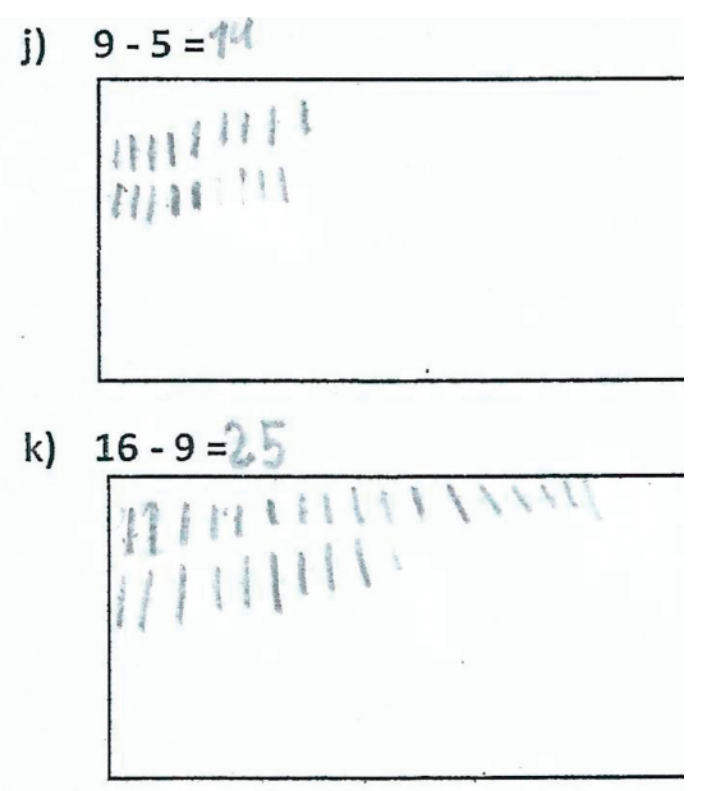

Figura 4. Resolución de operación de sustracción (Man, 9 años)

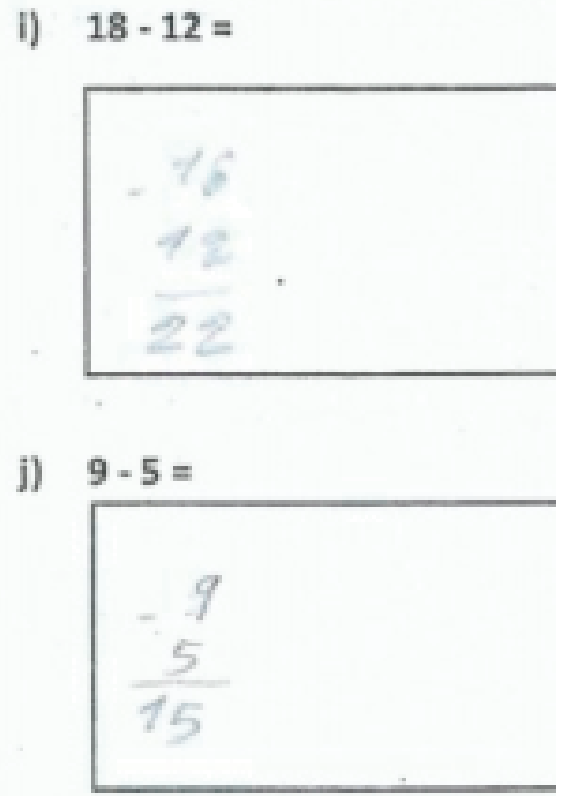

Figura 5. Resolución de operación de sustracción (Les, 9 años)

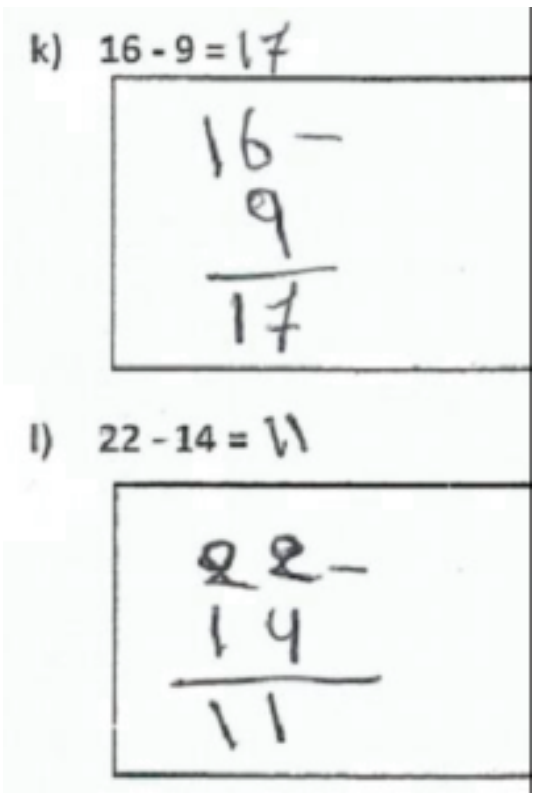

Figura 6. Resolución de operación de sustracción (Dal,11 años) 

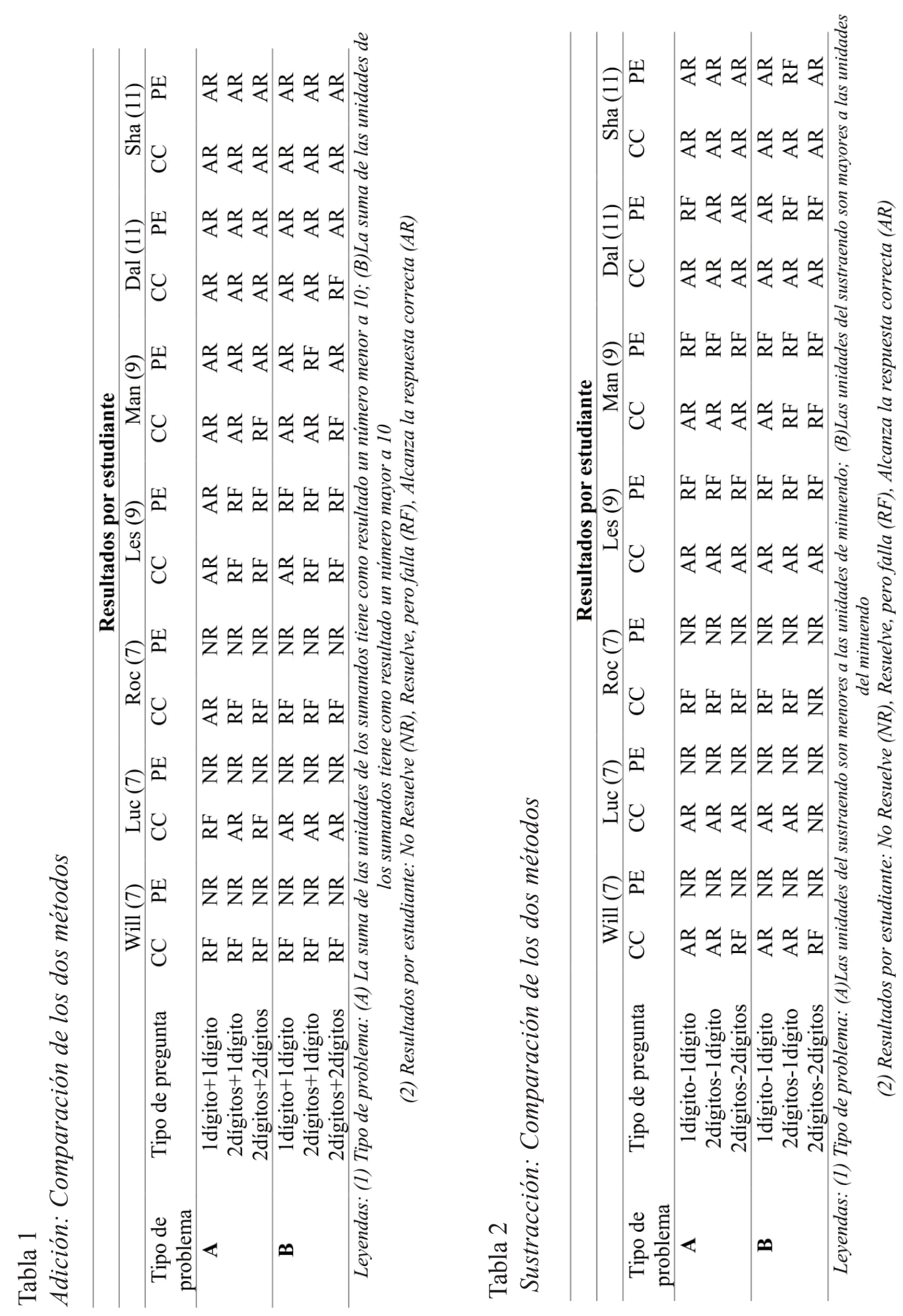


\section{Referencias bibliográficas}

Adams, J., Luitel, B., Afonso, E. \& Taylor, P. (2008). A cogenerative inquiry using postcolonial theory to envisage culturally inclusive science education. Cultural Studies of Science Education, 3(4), 999-1019. Recuperado el 1 de marzo de 2015 de http://dx.doi.org /10.1007/s11422-008-9130-0

Adjei, K. (1977). Influence of specific maternal occupation and behavior on Piagetian cognitive development. En P.R. Dasen (Ed.), Piagetian psychology: Cross-cultural contributions (pp. 227-256). New York: Gardner Press.

Aunio, P. \& Niemivirta, M. (2010). Predicting children's mathematical performance in grade one by early numeracy. Learning and Individual Differences, 20(5), 427-435. http:// dx.doi.org/10.1016/j.lindif.2010.06.003

Barrenechea, I. (2010). Evaluaciones estandarizadas: Seis reflexiones críticas [Standarized assessment: Six critical reflexions]. Education Policy Analysis Archives, 18(8), 1-25. Recuperado el 14 de abril de 2015 de http://www.redalyc.org/pdf/2750/2750197120 08.pdf

Becker, F. (2001) Educação e construção do conhecimento [Education and the construction of knowledge]. Sao Paulo: Artmed.

Becker, F. (2012). Epistemologia do professor de matemática [Epistemology of the math's teacher]. Petrópolis, RJ: Ed. Vozes.

Belaunde, L.E. (2012). Diseños materiales e inmateriales: La patrimonialización del kené shipibo-konibo y de la ayahuasca en el Perú [Material and immaterial designs: The patrimonialization of the Shipibo-Conibo kene designs and of the traditional use of ayahuasca in Peru]. Mundo Amazónico, 3, 123-146. Recuperado el 18 de mayo de 2015 de http://www.bdigital.unal.edu.co/29976/1/287 15-128077-2-PB.pdf http://dx.doi.org /10.5113/ma.3.28715

Bos, M.S., Ganimian, A.J. \& Vegas, E. (2013). América Latina en Pisa 2012. Brief \# 6:
¿Cómo se desempeñan los estudiantes pobres y ricos? [Latin America in Pisa 2012. Brief \# 6: How do poor and rich students perform?]. Washington, DC and Paris, France: InterAmerican Development Bank and Organization for Economic Co-operation and Development. Recuperado el 24 de marzo de 2015 de $\mathrm{http}: / /$ scholar.harvard.edu/files/alejandro_ganimian/files/brief_6_1.pdf

Bovet, M. (1974). Cognitive processes among illiterate children and adults. En J.W. Berry \& P.R. Dasen (Eds.), Culture and cognition: Readings in cross-cultural psychology. London: Methuen.

Brabec, B. \& Mori, L. (2009). La corona de la inspiración. Los diseños geométricos de los Shipibo-Konibo y sus relaciones con cosmovisión y música [The crown of inspiration. Shipibo-Konibo geometric designs and their relation to worldview and music]. Indiana, 26, 105-134. Recuperado el 9 de junio de $2015 \mathrm{de} \mathrm{http://neip.info/novo/wp-content/}$ uploads/2015/04/brabec_de_mori_corona-dela-inspiracion 2009.pdf

Bruner, J. (1966). Studies in cognitive growth. New York: Wiley.

Cáceres, L., De la Peña, J., Pineda, A., Di Prisco, C. \& Solotar, A. (2014). Mathematics in Latin America and the Caribbean: So much happening, so much to do. Recuperado el 23 de marzo de 2015 de http://www.ams.org/notices/201409/rnoti-p1052.pdf

Carnevale, A.P. (2005). Education and the economy: If we're so dumb, why are we so rich? Education Week, 24(21), 52-41.

Chavajay, P. \& Rogoff, B. (2002). Schooling and traditional collaborative social organization of problem solving by Mayan mothers and children. Developmental Psychology, 38(1), 55-66. Recuperado el 16 de julio de 2015 de https:// www.ncbi.nlm.nih.gov/pubmed/11806702 http://dx.doi.org/10.1037/0012-1649.38.1.55

Cueto, S. \& Díaz, J.J. (1999). Impacto de la educación inicial en el rendimiento en primer grado de primaria en escuelas públicas urba- 
nas de Lima [Impact of kindergarten education in 1st grade performance in urban public schools of the city of Lima]. Revista de Psicología de la PUCP, XVII(1), 73-91. Recuperado el 25 de agosto de 2015 de http://revistas.pucp.edu.pe/index.php/psicologia/article/view/7384/7605

Cueto, S., Guerrero, G. León, J. Zapata, M. \& Freire, S. (2013). ¿La cuna marca las oportunidades y el rendimiento educativo? Una mirada al caso peruano [Does students' background affect educational opportunities and outcomes? A look at the Peruvian case]. Recuperado el 9 de marzo de 2016 de http://repositorio.grade.org.pe/bitstream/GRA DE/61/1/ddt66.pdf

Cueto, S., Ramirez, C., León, J. \& Pain, O. (2003). Oportunidades de aprendizaje y rendimiento en matemáticas en una muestra de estudiantes del sexto grado de primaria de Lima [Learning opportunities and mathematics performance in sixth grade students from Lima city]. Documento de Investigación, 43. Lima: GRADE. Recuperado el 20 mayo de 2015 de http://repositorio.grade.org.pe/handle/ GRADE/84

Da Costa, L.D.F.M. \& de Lucena, I.C.R. (2015). Educação matemática na escola indígena: Implicações à formação de professores [Mathematics education at indigenous schools: Implications for teachers training]. Unión. Revista Iberoamericana de Educación Matemática, 44, 73-89. Recuperado el 17 de abril de 2015 de http://www.fisem.org/www/union/revistas/2015/44/20140708_Luc\%C3\% A9lida $\% 20$ de $\% 20 \mathrm{~F} \% \mathrm{C3} \%$ A1tima $\% 20$ Maia $\% 2$ $0 \mathrm{da} \% 20 \operatorname{Costa}(1) \cdot \mathrm{pdf}$

Dasen, P. (1984). The cross-cultural study of intelligence: Piaget and the Baoulé. International Journal of Psychology, 19, 407-434. Recuperado el 11 de junio de 2015 de http://dx.doi.org $/ 10.1080 / 00207598408247539$

Delval, J. (2001). Descubrir el pensamiento de los niños: Introducción a la práctica del método clínico-crítico [Discovering children's thinking: An introduction to the practice of the clinical-critical method]. Barcelona: Paidós.

Dewah, C. \& Van Wyk, M.M. (2014). The place of indigenous cultural games by educators in the teaching and learning of mathematics. Journal of Human Ecology, 48(1), 189-197. Recuperado el 26 de agosto de 2015 de http://www.krepublishers.com/02 Journals/JHE/JHE-48-0-000-14-Web/JHE48-1-000-14-Abst-PDF/JHE-48-1-189-14-26 85-Dewah-C/JHE-48-1-189-14-2685-DewahC-Tx[20].pdf

Dickerson, A. \& Popli, G. K. (2016). Persistent poverty and children's cognitive development: Evidence from the UK millennium cohort study. Journal of the Royal Statistical Society: Series A (Statistics in Society), 179(2), 535-558. Recuperado el 15 de septiembre de 2015 de http://onlinelibrary.wiley.com/doi/10.1111/rssa.12128/epdfhttp: //dx.doi.org/10.1111/rssa.12128

Dongo, A. (2002). Piaget y los niños marginados [Piaget and marginalized children]. Lima: Editora Vozes, Petrópolis.

Dongo, A. (2009). Significado de los factores sociales y culturales en el desarrollo cognitivo [Meaning of social and cultural factors in cognitive development]. Revista IIPSI, Facultad de Psicología de la Universidad Nacional Mayor de San Marcos, 12(2), 227-237. Recuperado el 14 de mayo de 2016 de http:// sisbib.unmsm.edu.pe/bvrevistas/investigacion_psicologia/v12_n2/pdf/a16v12N2.pdf

Dongo, A. (2011). Problemática psicosocial y educacional de los niños marginados. [Marginalized children's psychosocial and educational issues] Revista IIPSI, Facultad de Psicología de la Universidad Nacional Mayor de San Marcos, 14(1), 17-30. Recuperado el 8 de agosto de 2015 de http://sisbib.unmsm.edu.pe/ bvrevistas/investigacion_psicologia/v14_n1/pdf/a 02.pdf

Ducret, J.J. (2004). Methode clinique-critique Piagetienne [Piagetian clinical-critical method]. Genève: Service de la Recherché en Education. 
Flavell, J. (1976). La psicología evolutiva de Jean Piaget [The developmental pychology of Jean Piaget]. Buenos Aires: Paidós.

Frisancho, S., Delgado, E. \& Lam, L. (2015). El consentimiento informado en contextos de diversidad cultural: Trabajando en una comunidad Asháninka del Perú [Informed consent in culturally diverse contexts: Working in an Asháninka community in Peru]. Revista Interdisciplinaria de Filosofía y Psicología Limite, 10, 26-35. Recuperado el 25 de noviembre de 2015 dehttp://limite.uta.cl/index. php/limite/article/view/159/99

Fyhn, A.B., Jannok Nutti, Y. Nystad, K., Eira, L.J.S. \& Hætta, O.E. (2016). "We had not dared to do that earlier, but now we see that it works": Creating a culturally responsive mathematics exam. AlterNative:An International Journal of Indigenous Peoples, 12(4), 411424. http://dx.doi.org/10.20507/AlterNative. 2016.12.4.6

Galperin, Y. (1969). Stages in the development of mental acts. En M. Cole \& I. Maltzman (Eds.), A handbook of contemporary Soviet psychology (pp. 249-273). Nueva York: Basic Books.

Gascón, J. (2001). Incidencia del modelo epistemológico de las matemáticas sobre las prácticas docentes [The effect of mathematics epistemological model on teachers' practices]. Revista Latinoamericana de Investigación en Matemática Educativa, 4(2), 129-159. Recuperado el 23 de junio de 2015 de http://www.redalyc.org/pdf/335/33540202.pdf

Hallpike, C. (1986). Fundamentos del pensamiento primitivo [Foundations of primitive thought]. México: Fondo de Cultura Económica.

Ignat, S. \& Nataly, S. (2015). Influence of family education on the mental development of the child. Journal Plus Education / Educatia Plus, 13(2), 241-248. Recuperado el 17 de marzo de 2016 de http://www.uav.ro/jour/index. php/jpe /article/viewFile/ 589/649

Kamii, C. (1985). La teoría de Piaget en la edu- cación preescolar [Piaget theory for early education]. Madrid: Editorial Visor.

Kamii, C. (1994). Reinventando la aritmética III: Implicaciones de la teoría de Jean Piaget [To reinvent arithmetic III: Implications of Piaget's theory]. Madrid: Visor.

Kamii, C. (2000). El niño reinventa la aritmética: Implicaciones de la teoría de Piaget [Young children reinvent arithmetic: Implications of Piaget's theory]. Madrid: Visor.

Lacasa, P. (1989). Contexto y desarrollo cognitivo: Entrevista a Barbara Rogoff [Context and cognitive development: An interview with Barbara Rogoff]. Infancia y Aprendizaje, 45, 7-23. Recuperado el 18 de abril de 2015 de https://dialnet.unirioja.es/descarga/articulo /48318.pdf

Lakes, K.D., Vaughan, E., Jones, M., Burke, W., Baker, D. \& Swanson, J.M. (2012). Diverse perceptions of the informed consent process: Implications for the recruitment and participation of diverse communities in the national children's study. American Journal of Community Psychology, 49(1-2), 215-232. http://dx.doi.org/10.1007/s10464-011-9450-1

Lacunza, A., Contini, N. \& Castro, A. (2010). Las habilidades cognitivas en niños preescolares. Un estudio comparativo en un contexto de pobreza [Cognitive skills in pre-school children. A comparative study in a context of poverty]. Acta Colombiana de Psicología, 13(1), 25-34. Recuperado el 7 de agosto de 2015 de http://www.redalyc.org/articulo.oa?id=79815637 002

Lipina, S., Martelli, M.I., Vuelta, B., InjoqueRicle, I. \& Colombo, J. (2004). Pobreza y desempeño ejecutivo en alumnos preescolares de la Ciudad de Buenos Aires (República Argentina) [Poverty and cognitive functioning in preschool students of the City of Buenos Aires]. Interdisciplinaria, 21(2), 153-193. Recuperado el 28 de noviembre de 2016 de http://www.scielo.org.ar/pdf/interd/v21n2/v21 n2a02.pdf

Maynard, A. (2008). What we thought we knew and how we came to know it: Four decades of 
cross-cultural research from a Piagetian point of view. Human Development, 51, 56-65. http://dx.doi.org/10.1159/000113156

Meza A. \& Sirlopú D. (1997). La investigación psicológica peruana en temas piagetanos [Peruvian research on Piagetian topics]. En C. Thorne (Comp.), Piaget entre nosotros. Lima: PUCP.

Ministerio de Educación del Perú (MINEDU) (2009). Diseño curricular nacional de la educación básica regular [National curriculum program for basic education]. Lima: Minedu. Recuperado el 8 de febrero de 2015 de www.minedu.gob.pe/DeInteres/xtras/download.php?link=den_2009.pdf

Ministerio de Educación del Perú (MINEDU) (2011). Propuesta de diseño curricular diversificado con enfoque ambiental [Diversified curriculum proposal with environmental focus]. Ucayali: Dirección Regional de Educación de Ucayali.

Ministerio de Educación del Perú (MINEDU) (2013). Hacia una educación intercultural bilingüe de calidad. Propuesta pedagógica [Towards a quality bilingual intercultural education. A pedagogical proposal]. Recuperado el 19 de abril de 2015 de http://www.minedu.gob.pe/minedu/archivos/a/002/01-general/2-propuesta_pedaggogica_eib_2013.pdf

Ministerio de Cultura del Perú (2014). Bases de datos de pueblos indigenas $u$ originarios [Indigenous peoples data base]. Recuperado el 27 de agosto de 2015 de http://bdpi.cultura.gob.pe/pueblo/shipibo-konibo

Ministerio de Educación (2015). Rutas de aprendizaje: Versión 2015 [Paths to learning: Version 2015]. Lima: Ministerio de Educación del Perú. Recuperado el 9 de junio de 2015 de http://www.minedu.gob.pe/rutas-delaprendizaje/primaria.php

Ministerio de Educación (2016). Currículo Nacional de la Educación Básica [National school program for basic education]. Lima: Ministerio de Educación del Perú. Recuperado el 28 de mayo de 2016 de http:// www.minedu.gob.pe/curriculo/pdf/curriculonacional-2016.pdf

Mishra, R. (2014). Piagetian studies of cognitive development in India. Psychological Studies, 59(3), 207-222. http://dx.doi.org/10.1007/s 12646-014-0237-y

Monereo, C. (2014). Dime cómo evalúas y te diré cómo aprenden tus alumnos. El blog de educación y TIC. Recuperado el 26 de mayo de 2016 de http://blog.tiching.com/carles-monereo-dime-como-evaluas-y-te-dire-comoaprenden-tus-alumnos/

Morales, J.A. \& Frisancho, S. (2013). Operaciones combinatorias en estudiantes universitarios de ciclo inicial [Combinatorial operations in university freshmen]. Schème. Revista Electrónica de Psicología y Epistemología Genéticas, 5(2), 130-156. Recuperado el 16 de junio de 2015 de http:// www2.marilia. unesp.br/revistas/index.php/scheme/article/vi ew/3575/3731

Morales, J.A., Frisancho, S. \& Lam, L. (2016). Operación de combinatoria experimental en estudiantes que inician la educación universitaria [Experimental combinatorial operation in students entering university education]. Revista Electrónica de Enseñanza de las Ciencias 15, 1, 79-97. Recuperado el 5 de agosto de 2015 de http://reec.uvigo.es/volumenes/volumen15/REEC_15_1_5_ex976.pdf

Moreano, G., Asmad, U., Cruz, G. \& Cuglievan, G. (2008). Concepciones sobre la enseñanza de matemática en docentes de primaria de escuelas estatales [Conceptions about mathematic teaching in teachers of primary public shools]. Revista de Psicología, 26(2), 299 334. Recuperado el 10 de mayo de 2015 de http://revistas.pucp.edu.pe/index.php/psicologia/article/view/1064/1029

Murillo, J. (2007). Resultados de aprendizaje en América Latina a partir de las evaluaciones nacionales [Learning outcomes in Latin America based on national assessments]. Santiago de Chile: UNESCO/OREALC. Recuperado el 8 de junio de 2015 de http://unes- 
doc.unesco.org/images/0015/001555/155567 s.pdf

Musso, M. (2010). Funciones ejecutivas: Un estudio de los efectos de la pobreza sobre el desempeño ejecutivo [Executive Functions: A study about the impact of poverty on cognitive functioning]. Interdisciplinaria, 27(1), 95-110. Recuperado el 28 de noviembre de 2015 de http://www.scielo.org.ar/pdf/interd/ v27n1/v27n1a07.pdf

Nelson-Barber, S. \& Trumbull, E. (2007). Making assessment practices valid for indigenous american students. Journal of American Indian Education, 46(3), 132-147. Recuperado el 27 de junio de 2015 de https:// www.jstor.org/stable/pdf/24398547.pdf

Nunes, T. (1993). The socio-cultural context of mathematical thinking: Research findings and educational implications. En A.J. Bishop, K. Hart, S. Lerman \& T. Nunes (Eds.), Significant influences on children's learning of mathematics (pp. 27-42). París: UNESCO. Recuperado el 15 de abril de 2015 de http://www.unesco.org/education/pdf/323 47.pdf

Nunes, T. \& Bryant, P. (1996). Children doing mathematics (Understanding children's worlds). Oxford: Wiley-Blackwell.

Nunes, T., Carraher, D.W. \& Dias Schliemann, A. (1982). Na vida, dez; na escola, zero: Os contextos culturais da aprendizagem da matematica [In life ten, in school zero: The cultural context of mathematical learning]. Cadernos de Pesquisa São Paulo, 42, 79-86.

Opper, S. (1977). Concept development in Thai urban and rural children. En P.R. Dasen (Ed.), Piagetian psychology: Cross-cultural contributions. New York: Gardner Press.

Otero, I., Vizcaino, A. \& Carmenates, D. (2015). Creencias epistemológicas de profesores y alumnos sobre la matemática [Teachers and students' epistemological beliefs about mathematics]. Unión. Revista Iberoamericana de Educación Matemática, 42, 166-184. Recuperado el 23 de abril de 2016 de http:// www. fisem.org/www/union/revistas/2015/
42/42 Artigo8.pdf Recuperado el 3 de abril de 2015 de http://www.redalyc.org/ pdf/4030/ 403041701009.pdf

Paradise, R. (1991). El conocimiento cultural en el aula. Niños indígenas y su orientación hacia la observación [Cultural knowledge in the classroom: Indigenous children and their orientation towards observation]. Infancia $y$ Aprendizaje, 55, 73-85. https://dialnet.unirioja.es/descarga/articulo/48376.pdf

Paradise, R. (1996). Passivity or tacit collaboration: Mazahua interaction in cultural context. Learning and Instruction, 6(4), 379-389. Recuperado el 27 de junio de 2015 de http://www.sciencedirect.com/science/articlepii/S0959475296000229. http://dx.doi.org/ 10.1016/S0959-4752(96)00022-9

Paradise, R. \& Rogoff, B. (2009). Side by side: Learning by observing and pitching in. Ethos, 37(1), 102-138. Recuperado el 19 de agosto de 2015 de https://people.ucsc.edu/ brogoff/Scanned-articles/scanned \%2012 2008/Side\%20by\%20Side.pdf. http://dx. doi. org/10.1111/j.1548-1352.2009.01033.x

Parrat, S. (2016a). Conversaciones libres con los niños: El método clínico piagetiano. Relación entre teoría y método [Free conversations with children: Piagetian clinical method. The relation between theory and method]. En S. Frisancho (Coord.), Ensayos constructivistas (pp. 51-76). Lima: Fondo Editorial PUCP.

Parrat, S. (2016b). Sujeto-objeto-experimentador. Estrategias de intervención en situaciones causales [Subject-object-experimenter. Intervention strategies in causal situations]. En S. Frisancho (Coord.), Ensayos constructivistas (pp. 91-111). Lima: Fondo Editorial PUCP.

Pascual, L (2008). El contexto familiar y su acción mediadora en el desarrollo cognitivo y el rendimiento escolar [Family context and its mediating action in cognitive development and school performance]. Propuesta Educativa, 29(Junio), 73-82.

Piaget, J. (1971). Génesis del número en el niño [The child's conception of number]. Buenos 
Aires: Editorial Guadalupe.

Piaget, J. (1972). Psicología y epistemología [Psychology and epistemology: Towards a theory of knowledge]. París: Emencé Editores.

Piaget, J. (1975). Introducción a la epistemología genética. Tomo I: El pensamiento matemático [Introduction to genetic epistemology. Volume I: Mathematical thought]. Buenos Aires: Paidós.

Piaget, J. (1977a). Seis estudios de psicología [Six psychological studies]. Barcelona: Seix Barral.

Piaget, J. (1977). El juicio y el razonamiento en el niño: Estudio sobre la lógica del niño [Judgment and reasoning in the child]. Buenos Aires: Guadalupe.

Piaget, J. (1978). Investigaciones sobre la contradicción [Researchs in contradiction]. México: Siglo XXI Editores.

Piaget, J. \& García, R. (1982). Psicogénesis e historia de la ciencia [Psychogenesis and the history of science]. Madrid: Siglo XXI Editores.

Piaget, J. \& Inhelder, B. (1980). Psicología del niño [The psychology of the child]. Madrid: Morata.

Price-Williams, D.R. (1961). A study concerning concepts of conservation of quantities among primitive children. Acta Psychologica, 18, 297-305.http://dx.doi.org/10.1016/00016918(61)90019-1

Programme for International Student Assessment (PISA). (2012). Assessment framework. Key competencies in reading, mathematics and science. Recuperado el 18 de Febrero del 2015 de http://www.oecd.org/pisa/keyfindings/pisa-2012-results.htm

Ravela, P. (2002). ¿Cómo presentan sus resultados los sistemas nacionales de evaluación educativa en América Latina? [How do Latin America national educational evaluation systems present their results?]. Documento de Trabajo 22. Santiago: PREAL. Recuperado el 25 de octubre de 2015 de
http://baseddp.mec.gub.uy/Documentos/Bibliodi gi/PREAL\%2022.pdf

Rindermann, H. \& Baumeister, A.E. (2015). Parents' SES vs. parental educational behavior and children's development: A reanalysis of the Hart and Risley study. Learning and Individual Differences, 37133-37138. Recuperado el 29 de abril de 2016 de http://ac.elscdn.com/S1041608014002507/1-s2.0-S10416 08014002507-main.pdf?_tid=d9d1288eb 7cb-11e6-8649-00000aab0 f0 1\&acdnat $=1480599864 \_7187 \mathrm{~b} 14 \mathrm{e} 607862886762 \mathrm{cc}$ 92cd0dd5f0 http://dx.doi.org/10.1016/j.lindif.2014.12.005

Rogoff, B. (1993). Aprendices del pensamiento. El desarrollo cognitivo en el contexto social [Apprenticeship in thinking: Cognitive development in social context]. Barcelona: Ediciones Paidós.

Rogoff, B. (2003). The cultural nature of human development. New York: Oxford University Press.

SICRECE (2015). Sistema de consulta de resultados de la evaluación censal de estudiantes [Consultation system for students' census evaluation results]. Recuperado el 17 de enero de 2015 de http://sistemas02.minedu.gob.pe /consulta_ece/publico/index.php

Teixeira, J., Abud, S., Ribeiro, M. \& Fernandes, F. (2015). As concepções de álgebra e de educação algébrica - uma análise de livros didáticos do $8^{\circ}$ ano [Conceptions of algebra and algebra education: An analysis of didactic books for 8th grade]. Revista Profissão Docente Uberaba, 15(33), 127-145. Recuperado el 12 de enero de 2016 de http:// www.revistas.uniube.br/index.php/rpd/article/view/1 014/1197

Treviño, E. (2006). Evaluación del aprendizaje de los estudiantes indígenas en América Latina. Desafíos de medición e interpretación en contextos de diversidad cultural y desigualdad social [Learning evaluation of indigenous students in Latin America. Measurement and interpretation challenges in 
culturally diverse and socially unequal contexts]. Revista Mexicana de Investigación Educativa, Enero-Marzo, 225-268. Recuperado el 14 de mayo de 2015 de http://www.redalyc.org/pdf/140/14002812.pdf

Tillema, E.S. (2012). What is the difference? Using contextualized problems. Mathematics Teaching in the Middle School, 17(8), 472-478.

UNESCO. (2014). Tercer estudio regional comparativo y explicativo [Third regional comparative and explanatory study]. Santiago de Chile: Oficina Regional de Educación para América Latina y el Caribe. Recuperado el 27 de agosto de 2015 de http://www.unesco.org /new/fileadmin/MULTIMEDIA/FIELD/Santi ago/pdf/Kit_TERCE.pdf

Unidad de Medición de la Calidad Educativa (UMC). (2013). Resultados de la evaluación Censal de Estudiantes 2013 [Results of students' census 2013]. Recuperado el 16 de noviembre de 2015 de http:/umc.minedu.gob.pe/evaluacion-censal-de-estudiantes-2013-ece-2013

Unidad de Medición de la Calidad Educativa (UMC). (2014). Resultados de la evaluación Censal de Estudiantes 2014 [Results of students' census 2014]. Recuperado el 16 de noviembre de 2015 de http://umc.minedu.gob.pe/evaluacion-censal-de-estudiantes-2014-ece-2014

Unidad de Medición de la Calidad Educativa (UMC). (2015). Resultados de la evaluación Censal de Estudiantes 2015 [Results of students' census 2015]. Recuperado el 16 de noviembre de 2015 de http://umc.minedu.gob.pe /evaluacion-censal-de-estudiantes-ece-2015

Valverde, G. \& Näslund-Hadley, E. (2010). La condición de la educación en matemáticas y ciencias naturales en América Latina y el Caribe [The state of numeracy education in Latin America and the Caribbean]. BID, Banco Interamericano de Desarrollo. Recuperado el 16 de noviembre de 2015 de http://www.oei.es/salactsi/bidciencias.pdf

Vygotsky, L.S. (1983). Obras escogidas. Tomo III. Problemas del desarrollo de la psique [Selected writtings. Volume III. Problems in the development of the mind]. Madrid: Visor.

Wolfgang, C.H., Stannard, L.L. \& Jones, I. (2001). Block play performance among preschoolers as a predictor of later school achievement in mathematics. Journal of Research in Childhood Education, 15(2), 173-180. Recuperado el 13 de enero de 2015 de http://www.tandfonline.com/doi/abs/10.1080/ 02568540109594958? needAccess $=$ true \#aHR 0cDovL3d3dy50YW5kZm9ubGluZS5jb20v ZG9pL3BkZi8xMC4xMDgwLzAyNTY4NT QwMTA5NTk0OTU4P251ZWRBY2Nlc3M9 dHJ1ZUBAQDA=http://dx.doi.org/10.1080/0 2568540109594958

Woolfolk, A. (2010). Psicología educativa [Educational psychology]. México: Pearson.

Zavala, V. (2012). Dilemas ideológicos en torno a la educación intercultural bilingüe: El caso de la lectura en quechua [Ideological dilemmas around intercultural bilingual education: The case of reading in Quechua]. Revista Peruana de Investigación Educativa, 4, 77-104. Recuperado el 15 de noviembre de 2015 de http://siep.org.pe/ wp-content/uploads/264.pdf

Fecha de recepción: $1^{\circ}$ de octubre de 2016 Fecha de aceptación: 29 de marzo de 2017 